\title{
Oral Rehydration Solution OS-1
}

National Cancer Institute

\section{Source}

National Cancer Institute. Oral Rehydration Solution OS-1. NCI Thesaurus. Code C111035.

An aqueous solution composed of glucose and electrolytes, including sodium, potassium, chloride, magnesium, and phosphorus, with dehydration preventative and rehydration activities. Upon oral administration of the oral rehydration solution (ORS), water, electrolytes and glucose are absorbed from the gastrointestinal (Gl) tract into the systemic circulation. This replenishes the body's supply of water, carbohydrates and electrolytes, and prevents both dehydration and renal dysfunction. 